\title{
Existence of Solutions for Nonlocal Boundary Value Problems of Higher-Order Nonlinear Fractional Differential Equations
}

\author{
Bashir Ahmad $^{1}$ and Juan J. Nieto ${ }^{2}$ \\ ${ }^{1}$ Department of Mathematics, Faculty of Science, King Abdulaziz University, P.O. Box 80203, \\ Jeddah 21589, Saudi Arabia \\ ${ }^{2}$ Departamento de Análisis Matemático, Facultad de Matemáticas, Universidad de Santiago de Compostela, \\ 15782 Santiago de Compostela, Spain
}

Correspondence should be addressed to Bashir Ahmad, bashir_qau@yahoo.com

Received 19 February 2009; Accepted 27 April 2009

Recommended by Paul Eloe

We study some existence results in a Banach space for a nonlocal boundary value problem involving a nonlinear differential equation of fractional order $q$ given by ${ }^{c} D^{q} x(t)=f(t, x(t))$, $0<t<1, q \in(m-1, m], m \in \mathbb{N}, m \geq 2, x(0)=0, x^{\prime}(0)=0, x^{\prime \prime}(0)=0, \ldots, x^{(m-2)}(0)=0$, $x(1)=\alpha x(\eta)$. Our results are based on the contraction mapping principle and Krasnoselskii's fixed point theorem.

Copyright (C) 2009 B. Ahmad and J. J. Nieto. This is an open access article distributed under the Creative Commons Attribution License, which permits unrestricted use, distribution, and reproduction in any medium, provided the original work is properly cited.

\section{Introduction}

Fractional differential equations involve derivatives of fractional order. They arise in many engineering and scientific disciplines such as the mathematical modeling of systems and processes in the fields of physics, chemistry, aerodynamics, electro-dynamics of complex medium, and polymer rheology. In consequence, the subject of fractional differential equations is gaining much importance and attention. For examples and details, see [1-17] and the references therein. However, the theory of boundary value problems for nonlinear fractional differential equations is still in the initial stages and many aspects of this theory need to be explored.

The subject of multipoint nonlocal boundary value problems, initiated by Ilin and Moiseev [18, 19], has been addressed by many authors, for instance, [20-26]. The multipoint boundary conditions appear in certain problems of thermodynamics, elasticity, and wave propagation, see [27] and the references therein. The multipoint boundary conditions may be understood in the sense that the controllers at the end points dissipate or add energy according to censors located at intermediate positions. 
For $m \in \mathbb{N}, m \geq 2$, and $q \in(m-1, m]$, we consider the following nonlinear fractional differential equation of order $q$ with nonlocal boundary conditions:

$$
\begin{gathered}
{ }^{c} D^{q} x(t)=f(t,(t)), \quad 0<t<1, \\
x(0)=0, \quad x^{\prime}(0)=0, \quad x^{\prime \prime}(0)=0, \ldots, x^{(m-2)}(0)=0, \quad x(1)=\alpha x(\eta), \\
0<\eta<1, \quad \alpha \eta^{m-1} \neq 1, \quad \alpha \in \mathbb{R},
\end{gathered}
$$

where ${ }^{c} D$ is the Caputo fractional derivative and $f:[0,1] \times X \rightarrow X$ is continuous. Here, $(X,\|\cdot\|)$ is a Banach space and $C=C([0,1], X)$ denotes the Banach space of all continuous functions from $[0,1] \rightarrow X$ endowed with a topology of uniform convergence with the norm denoted by $\|\cdot\|$.

By a solution of (1.1), we mean a function $x \in \mathcal{C}$ of class $C^{m}[0,1]$ which satisfies the nonlocal fractional boundary value problem (1.1).

\section{Preliminaries}

Let us recall some basic definitions $[12,15,17]$ on fractional calculus.

Definition 2.1. For a function $g:[0, \infty) \rightarrow \mathbb{R}$, the Caputo derivative of fractional order $q$ is defined as

$$
{ }^{c} D^{q} g(t)=\frac{1}{\Gamma(n-q)} \int_{0}^{t}(t-s)^{n-q-1} g^{(n)}(s) d s, \quad n-1<q<n, n=[q]+1,
$$

where $[q]$ denotes the integer part of the real number $q$.

Definition 2.2. The Riemann-Liouville fractional integral of order $q$ is defined as

$$
I^{q} g(t)=\frac{1}{\Gamma(q)} \int_{0}^{t} \frac{g(s)}{(t-s)^{1-q}} d s, \quad q>0
$$

provided that the integral exists.

Definition 2.3. The Riemann-Liouville fractional derivative of order $q$ for a function $g(t)$ is defined by

$$
D^{q} g(t)=\frac{1}{\Gamma(n-q)}\left(\frac{d}{d t}\right)^{n} \int_{0}^{t} \frac{g(s)}{(t-s)^{q-n+1}} d s, \quad n=[q]+1
$$

provided the right hand side is pointwise defined on $(0, \infty)$.

We remark that the Caputo derivative becomes the conventional $n$th derivative of the function as $q \rightarrow n$ and the initial conditions for fractional differential equations retain the same form as that of ordinary differential equations with integer-order derivatives. On the other hand, the Riemann-Liouville fractional derivative could hardly produce the physical interpretation of the initial conditions required for the initial value problems 
involving fractional differential equations (the same applies to the boundary value problems of fractional differential equations). Moreover, the Caputo derivative for a constant is zero while the Riemann-Liouville fractional derivative of a constant is nonzero. For more details, see [17].

Lemma 2.4 (see [28]). For $q>0$, the general solution of the fractional differential equation ${ }^{c} D^{q} x(t)=0$ is given by

$$
x(t)=c_{0}+c_{1} t+c_{2} t^{2}+\cdots+c_{n-1} t^{n-1},
$$

where $c_{i} \in \mathbb{R}, i=0,1,2, \ldots, n-1(n=[q]+1)$.

In view of Lemma 2.4, it follows that

$$
I^{q c} D^{q} x(t)=x(t)+c_{0}+c_{1} t+c_{2} t^{2}+\cdots+c_{n-1} t^{n-1},
$$

for some $c_{i} \in \mathbb{R}, i=0,1,2, \ldots, n-1(n=[q]+1)$.

Now, we state a known result due to Krasnoselskii [29] which is needed to prove the existence of at least one solution of (1.1).

Theorem 2.5. Let $M$ be a closed convex and nonempty subset of a Banach space X. Let $A, B$ be the operators such that

(i) $A x+B y \in M$ whenever $x, y \in M$,

(ii) $A$ is compact and continuous,

(iii) $B$ is a contraction mapping.

Then there exists $z \in M$ such that $z=A z+B z$.

To study the nonlinear problem (1.1), we first consider the associated linear problem and obtain its solution.

Lemma 2.6. For a given $\sigma \in C[0,1]$, the unique solution of the boundary value problem,

$$
\begin{gathered}
{ }^{c} D^{q} x(t)=\sigma(t), \quad 0<t<1, \quad q \in(m-1, m], \quad m \in \mathbb{N}, \quad m \geq 2, \\
x(0)=0, \quad x^{\prime}(0)=0, \quad x^{\prime \prime}(0)=0, \ldots, x^{(m-2)}(0)=0, \quad x(1)=\alpha x(\eta), \\
0<\eta<1, \quad \alpha \eta^{m-1} \neq 1, \quad \alpha \in \mathbb{R},
\end{gathered}
$$

is given by

$$
x(t)=\int_{0}^{t} \frac{(t-s)^{q-1}}{\Gamma(q)} \sigma(s) d s-\frac{t^{m-1}}{\left(1-\alpha \eta^{m-1}\right)}\left[\int_{0}^{1} \frac{(1-s)^{q-1}}{\Gamma(q)} \sigma(s) d s-\alpha \int_{0}^{\eta} \frac{(\eta-s)^{q-1}}{\Gamma(q)} \sigma(s) d s\right] .
$$


Proof. Using (2.5), we have

$$
x(t)=\int_{0}^{t} \frac{(t-s)^{q-1}}{\Gamma(q)} \sigma(s) d s-c_{0}-c_{1} t-c_{2} t^{2}-\cdots-c_{m-1} t^{m-1},
$$

where $c_{0}, c_{1}, c_{2}, \ldots, c_{m-1} \in \mathbb{R}$ are arbitrary constants. In view of the relations ${ }^{c} D^{q} I^{q} x(t)=x(t)$ and $I^{q} I^{p} x(t)=I^{q+p} x(t)$ for $q, p>0, x \in L(0,1)$, we obtain

$$
\begin{aligned}
& x^{\prime}(t)=\int_{0}^{t} \frac{(t-s)^{q-2}}{\Gamma(q-1)} \sigma(s) d s-c_{1}-2 c_{2} t-\cdots-(m-1) c_{m-1} t^{m-2}, \\
& x^{\prime \prime}(t)=\int_{0}^{t} \frac{(t-s)^{q-3}}{\Gamma(q-2)} \sigma(s) d s-2 c_{2}-\cdots-(m-1)(m-2) c_{m-1} t^{m-3}, \ldots
\end{aligned}
$$

Applying the boundary conditions for (2.6), we find that $c_{0}=0, c_{1}=0, \ldots, c_{m-2}=0$, and

$$
c_{m-1}=\frac{1}{\left(1-\alpha \eta^{m-1}\right)}\left[\int_{0}^{1} \frac{(1-s)^{q-1}}{\Gamma(q)} \sigma(s) d s-\alpha \int_{0}^{\eta} \frac{(\eta-s)^{q-1}}{\Gamma(q)} \sigma(s) d s\right] .
$$

Substituting the values of $c_{0}, c_{1}, \ldots, c_{m-1}$ in (2.8), we obtain

$$
\begin{aligned}
x(t)= & \int_{0}^{t} \frac{(t-s)^{q-1}}{\Gamma(q)} \sigma(s) d s \\
& -\frac{t^{m-1}}{\left(1-\alpha \eta^{m-1}\right)}\left[\int_{0}^{1} \frac{(1-s)^{q-1}}{\Gamma(q)} \sigma(s) d s-\alpha \int_{0}^{\eta} \frac{(\eta-s)^{q-1}}{\Gamma(q)} \sigma(s) d s\right] .
\end{aligned}
$$

This completes the proof.

\section{Main Results}

For the forthcoming analysis, we need the following assumptions:

$\left(\mathrm{A}_{1}\right)\|f(t, x)-f(t, y)\| \leq L\|x-y\|$, forall $t \in[0,1], x, y \in X ;$

$\left(\mathrm{A}_{2}\right)\|f(t, x)\| \leq \mu(t)$, forall $(t, x) \in[0,1] \times X, \mu \in L^{1}\left([0,1], R^{+}\right)$.

In relation to the nonlocal problem (1.1), we define the constants:

$$
\Lambda=\frac{L}{\Gamma(q+1)}+\lambda, \quad \lambda=\frac{L\left(1+|\alpha| \eta^{q}\right)}{\Gamma(q+1)\left|1-\alpha \eta^{m-1}\right|}
$$

Theorem 3.1. Assume that $f:[0,1] \times X \rightarrow X$ is a jointly continuous function and satisfies the assumption $\left(A_{1}\right)$. Then the boundary value problem (1.1) has a unique solution provided $\Lambda<1$, where $\Lambda$ is given by (3.1). 
Proof. Define $\digamma: \mathcal{C} \rightarrow \mathcal{C}$ by

$$
\begin{aligned}
(\digamma x)(t)=\int_{0}^{t} & \frac{(t-s)^{q-1}}{\Gamma(q)} f(s, x(s)) d s-\frac{t^{m-1}}{\left(1-\alpha \eta^{m-1}\right)} \\
\times & {\left[\int_{0}^{1} \frac{(1-s)^{q-1}}{\Gamma(q)} f(s, x(s)) d s-\alpha \int_{0}^{\eta} \frac{(\eta-s)^{q-1}}{\Gamma(q)} f(s, x(s)) d s\right], \quad t \in[0,1] . }
\end{aligned}
$$

Let us set $\sup _{t \in[0,1]}\|f(t, 0)\|=M$, and choose

$$
r \geq \frac{M}{(1-\beta) \Gamma(q+1)}\left(1+\frac{1+|\alpha| \eta^{q}}{\left|1-\alpha \eta^{m-1}\right|}\right)
$$

where $\beta$ is such that $\Lambda \leq \beta<1$. Now we show that $\digamma B_{r} \subset B_{r}$, where $B_{r}=\{x \in \mathcal{C}:\|x\| \leq r\}$. For $x \in B_{r}$, we have

$$
\begin{aligned}
\|(\digamma x)(t)\| \leq & \int_{0}^{t} \frac{(t-s)^{q-1}}{\Gamma(q)}\|f(s, x(s))\| d s \\
& +\frac{t^{m-1}}{\left|1-\alpha \eta^{m-1}\right|}\left[\int_{0}^{1} \frac{(1-s)^{q-1}}{\Gamma(q)}\|f(s, x(s))\| d s+|\alpha| \int_{0}^{\eta} \frac{(\eta-s)^{q-1}}{\Gamma(q)}\|f(s, x(s))\| d s\right] \\
\leq & \int_{0}^{t} \frac{(t-s)^{q-1}}{\Gamma(q)}(\|f(s, x(s))-f(s, 0)\|+\|f(s, 0)\|) d s \\
& +\frac{t^{m-1}}{\left|1-\alpha \eta^{m-1}\right|}\left[\int_{0}^{1} \frac{(1-s)^{q-1}}{\Gamma(q)}(\|f(s, x(s))-f(s, 0)\|+\|f(s, 0)\|) d s\right. \\
\leq & (L r+M)\left(\int_{0}^{t} \frac{(t-s)^{q-1}}{\Gamma(q)} d s+\frac{t^{m-1}}{\left|1-\alpha \eta^{m-1}\right|}\left[\int_{0}^{1} \frac{(1-s)^{q-1}}{\Gamma(q)} d s+|\alpha| \int_{0}^{\eta} \frac{(\eta-s)^{q-1}}{\Gamma(q)} d s\right]\right) \\
\leq & \left(\frac{L}{\Gamma(q+1)}+\frac{L\left(1+|\alpha| \eta^{q}\right)}{\Gamma(q+1)\left|1-\alpha \eta^{m-1}\right|}\right) r+\frac{M}{\Gamma(q+1)}\left(1+\frac{1+|\alpha| \eta^{q}}{\left|1-\alpha \eta^{m-1}\right|}\right) \\
= & \Lambda r+\frac{M}{\Gamma(q+1)}\left(1+\frac{1+|\alpha| \eta^{q}}{\left|1-\alpha \eta^{m-1}\right|}\right) \\
\leq & (\Lambda+1-\beta) r \leq r .
\end{aligned}
$$


Now, for $x, y \in \mathcal{C}$ and for each $t \in[0,1]$, we obtain

$$
\begin{aligned}
& \|(\digamma x)(t)-(\digamma y)(t)\| \\
& \leq \int_{0}^{t} \frac{(t-s)^{q-1}}{\Gamma(q)}\|f(s, x(s))-f(s, y(s))\| d s \\
& \quad+\frac{t^{m-1}}{\left|1-\alpha \eta^{m-1}\right|}\left[\int_{0}^{1} \frac{(1-s)^{q-1}}{\Gamma(q)}\|f(s, x(s))-f(s, y(s))\| d s\right. \\
& \leq L\|x-y\|\left(\int_{0}^{t} \frac{(t-s)^{q-1}}{\Gamma(q)} d s+\frac{t^{m-1}}{\left|1-\alpha \eta^{m-1}\right|}\left[\int_{0}^{1} \frac{(1-s)^{q-1}}{\Gamma(q)} d s+|\alpha| \int_{0}^{\eta} \frac{(\eta-s)^{q-1}}{\Gamma(q)} d s\right]\right) \\
& =L\|x-y\|\left(\frac{t^{q}}{\Gamma(q+1)}+\frac{t^{m-1}}{\left|1-\alpha \eta^{m-1}\right|}\left(\frac{1+|\alpha| \eta^{q}}{\Gamma(q+1)}\right)\right) \\
& \leq L\|x-y\| \frac{1}{\Gamma(q+1)}\left(1+\frac{1+|\alpha| \eta^{q}}{\left|1-\alpha \eta^{m-1}\right|}\right) \\
& =\Lambda\|x-y\| .
\end{aligned}
$$

Clearly $\Lambda$ depends on the parameters $(q, m, \alpha, \eta, L)$ involved in the problem. As $\Lambda<1$, therefore, $\digamma$ is a contraction. Thus, the conclusion of the theorem follows by the contraction mapping principle.

Theorem 3.2. Let $f:[0,1] \times X \rightarrow X$ be a jointly continuous function mapping bounded subsets of $[0,1] \times X$ into relatively compact subsets of X. Further, the assumptions $\left(A_{1}\right)-\left(A_{2}\right)$ hold with $\lambda<1$, where $\lambda$ is given by (3.1). Then the boundary value problem (1.1) has at least one solution on $[0,1]$.

Proof. Let us fix

$$
r \geq \frac{\|\mu\|_{L^{1}}}{\Gamma(q)}\left(1+\frac{1+|\alpha| \eta^{q-1}}{\left|1-\alpha \eta^{m-1}\right|}\right)
$$

and consider $B_{r}=\{x \in \mathcal{C}:\|x\| \leq r\}$. We define the operators $\Phi$ and $\Psi$ on $B_{r}$ as

$$
\begin{aligned}
& (\Phi x)(t)=\frac{1}{\Gamma(q)} \int_{0}^{t}(t-s)^{q-1} f(s, x(s)) d s \\
& (\Psi x)(t)=-\frac{t^{m-1}}{\left(1-\alpha \eta^{m-1}\right)}\left[\int_{0}^{1} \frac{(1-s)^{q-1}}{\Gamma(q)} f(s, x(s)) d s-\alpha \int_{0}^{\eta} \frac{(\eta-s)^{q-1}}{\Gamma(q)} f(s, x(s)) d s\right] .
\end{aligned}
$$


For $x, y \in B_{r}$, we find that

$$
\|\Phi x+\Psi y\| \leq \frac{\|\mu\|_{L^{1}}}{\Gamma(q)}\left(1+\frac{1+|\alpha| \eta^{q-1}}{\left|1-\alpha \eta^{m-1}\right|}\right) \leq r .
$$

Thus, $\Phi x+\Psi y \in B_{r}$. It follows from the assumption $\left(A_{1}\right)$ that $\Psi$ is a contraction mapping for $\lambda<1$. Continuity of $f$ implies that the operator $\Phi$ is continuous. Also, $\Phi$ is uniformly bounded on $B_{r}$ as

$$
\|\Phi x\| \leq \frac{\|\mu\|_{L^{1}}}{\Gamma(q)}
$$

To show that the operator $\Phi$ is compact, we use the classical Arzela-Ascoli theorem. Let $\mathcal{A}$ be a bounded subset of $\mathcal{C}$. We have to show that $\Phi(\mathcal{A})$ is equicontinuous and for each $t$, the set $\Phi(\mathcal{A})(t)$ is relatively compact in $X$. In view of $\left(A_{1}\right),\left(A_{2}\right)$, we define $\sup _{(t, x) \in[0,1] \times B_{r}}\|f(t, x)\|=$ $f_{\max }$ and consequently we have

$$
\begin{aligned}
& \left\|(\Phi x)\left(t_{1}\right)-(\Phi x)\left(t_{2}\right)\right\| \\
& =\left\|\frac{1}{\Gamma(q)} \int_{0}^{t_{1}}\left(\left(t_{2}-s\right)^{q-1}-\left(t_{1}-s\right)^{q-1}\right) f(s, x(s)) d s+\int_{t_{1}}^{t_{2}}\left(t_{2}-s\right)^{q-1} f(s, x(s)) d s\right\| \\
& \leq \frac{f_{\max }}{\Gamma(q+1)}\left|2\left(t_{2}-t_{1}\right)^{q}+t_{1}^{q}-t_{2}^{q}\right|,
\end{aligned}
$$

which is independent of $x$. Thus, $\Phi$ is equicontinuous. Using the fact that $f$ maps bounded subsets into relatively compact subsets, we have that $\Phi(\mathcal{A})(t)$ is relatively compact in $X$ for every $t$. Therefore, $\Phi$ is relatively compact on $B_{r}$. Hence, By Arzela-Ascoli theorem, $\Phi$ is compact on $B_{r}$. Thus all the assumptions of Theorem 2.5 are satisfied and the conclusion of Theorem 2.5 implies that the boundary value problem (1.1) has at least one solution on $[0,1]$.

Example 3.3. Consider the following boundary value problem:

$$
\begin{gathered}
{ }^{c} D^{q} x(t)=\frac{1}{(t+7)^{2}} \frac{\|x\|}{1+\|x\|}, \quad 2<q \leq 3, t \in[0,1], \\
x(0)=0, \quad x^{\prime}(0)=0, \quad x(1)=x\left(\frac{1}{2}\right) .
\end{gathered}
$$

Here, $m=3, f(t, x(t))=\left(1 /(t+7)^{2}\right)(\|x\| /(1+\|x\|)), \alpha=1, \eta=1 / 2$. As $\|f(t, x)-f(t, y)\| \leq$ $(1 / 49)\|x-y\|$, therefore, $\left(A_{1}\right)$ is satisfied with $L=1 / 49$. Further,

$$
\Lambda=\frac{L}{\Gamma(q+1)}\left(1+\frac{1+|\alpha| \eta^{q}}{\left|1-\eta^{m-1}\right|}\right)=\frac{1}{49 \Gamma(q+1)}\left(1+\frac{4}{3}\left(1+\left(\frac{1}{2}\right)^{q}\right)\right)<1, \quad 2<q \leq 3
$$

Thus, by Theorem 3.1, the boundary value problem (3.11) has a unique solution on $[0,1]$. 


\section{Acknowledgments}

The authors thank the reviewers for their useful comments. The research of J. J. Nieto has been partially supported by Ministerio de Educacion y Ciencia and FEDER, project MTM200761724, and by Xunta de Galicia and FEDER, project PGIDIT06PXIB207023PR.

\section{References}

[1] B. Ahmad and J. J. Nieto, "Existence results for a coupled system of nonlinear functional differential equation with three-point boundary value problem," preprint.

[2] B. Ahmad and J. J. Nieto, "Existence results for nonlinear boundary value problems of fractional integrodifferential equations with integral boundary conditions," Boundary Value Problems, vol. 2009, Article ID 708576, 11 pages, 2009.

[3] B. Ahmad and V. Otero-Espinar, "Existence of solutions for fractional differential inclusions with antiperiodic boundary conditions," Article ID 625347, Boundary Value Problems. In press.

[4] B. Ahmad and S. Sivasundaram, "Existence and uniqueness results for nonlinear boundary value problems of fractional differential equations with separated boundary conditions," Communications in Applied Analysis, vol. 13, pp. 121-228, 2009.

[5] J. Allison and N. Kosmatov, "Multi-point boundary value problems of fractional order," Communications in Applied Analysis, vol. 12, pp. 451-458, 2008.

[6] D. Araya and C. Lizama, "Almost automorphic mild solutions to fractional differential equations," Nonlinear Analysis: Theory, Methods \& Applications, vol. 69, no. 11, pp. 3692-3705, 2008.

[7] B. Bonilla, M. Rivero, L. Rodríguez-Germá, and J. J. Trujillo, "Fractional differential equations as alternative models to nonlinear differential equations," Applied Mathematics and Computation, vol. 187, no. 1, pp. 79-88, 2007.

[8] Y.-K. Chang and J. J. Nieto, "Some new existence results for fractional differential inclusions with boundary conditions," Mathematical and Computer Modelling, vol. 49, no. 3-4, pp. 605-609, 2009.

[9] V. Gafiychuk, B. Datsko, and V. Meleshko, "Mathematical modeling of time fractional reactiondiffusion systems," Journal of Computational and Applied Mathematics, vol. 220, no. 1-2, pp. 215-225, 2008.

[10] V. Daftardar-Gejji and S. Bhalekar, "Boundary value problems for multi-term fractional differential equations," Journal of Mathematical Analysis and Applications, vol. 345, no. 2, pp. 754-765, 2008.

[11] R. W. Ibrahim and M. Darus, "Subordination and superordination for univalent solutions for fractional differential equations," Journal of Mathematical Analysis and Applications, vol. 345, no. 2, pp. 871-879, 2008.

[12] A. A. Kilbas, H. M. Srivastava, and J. J. Trujillo, Theory and Applications of Fractional Differential Equations, vol. 204 of North-Holland Mathematics Studies, Elsevier Science, Amsterdam, The Netherlands, 2006.

[13] S. Ladaci, J. J. Loiseau, and A. Charef, "Fractional order adaptive high-gain controllers for a class of linear systems," Communications in Nonlinear Science and Numerical Simulation, vol. 13, no. 4, pp. 707-714, 2008.

[14] M. P. Lazarević, "Finite time stability analysis of $\mathrm{PD}^{\alpha}$ fractional control of robotic time-delay systems," Mechanics Research Communications, vol. 33, no. 2, pp. 269-279, 2006.

[15] I. Podlubny, Fractional Differential Equations, vol. 198 of Mathematics in Science and Engineering, Academic Press, San Diego, Calif, USA, 1999.

[16] S. Z. Rida, H. M. El-Sherbiny, and A. A. M. Arafa, "On the solution of the fractional nonlinear Schrödinger equation," Physics Letters A, vol. 372, no. 5, pp. 553-558, 2008.

[17] S. G. Samko, A. A. Kilbas, and O. I. Marichev, Fractional Integrals and Derivatives: Theory and Applications, Gordon and Breach Science, Yverdon, Switzerland, 1993.

[18] V. A. Ilin and E. I. Moiseev, "Nonlocal boundary value problem of the first kind for a Sturm Liouville operator in its differential and finite difference aspects," Differential Equations, vol. 23, pp. 803-810, 1987.

[19] V. A. Ilin and E. I. Moiseev, "Nonlocal boundary value problem of the second kind for a Sturm Liouville operator," Differential Equations, vol. 23, pp. 979-987, 1987.

[20] B. Ahmad, "Approximation of solutions of the forced Duffing equation with $m$-point boundary conditions," Communications in Applied Analysis, vol. 13, pp. 11-20, 2009. 
[21] Y.-K. Chang, J. J. Nieto, and W.-S. Li, “On impulsive hyperbolic differential inclusions with nonlocal initial conditions," Journal of Optimization Theory and Applications, vol. 140, no. 3, pp. 431-442, 2009.

[22] Y.-K. Chang, J. J. Nieto, and W. S. Li, "Controllability of semi-linear differential systems with nonlocal initial conditions in Banach spaces," inpress Journal of Optimization Theory and Applications.

[23] P. W. Eloe and B. Ahmad, "Positive solutions of a nonlinear $n$th order boundary value problem with nonlocal conditions," Applied Mathematics Letters, vol. 18, no. 5, pp. 521-527, 2005.

[24] J. R. Graef and J. R. L. Webb, "Third order boundary value problems with nonlocal boundary conditions," Nonlinear Analysis: Theory, Methods \& Applications, vol. 71, no. 5-6, pp. 1542-1551, 2009.

[25] P. Gurevich, "Smoothness of generalized solutions for higher-order elliptic equations with nonlocal boundary conditions," Journal of Differential Equations, vol. 245, no. 5, pp. 1323-1355, 2008.

[26] R. Ma, "Positive solutions of a nonlinear $m$-point boundary value problem," Computers \& Mathematics with Applications, vol. 42, no. 6-7, pp. 755-765, 2001.

[27] R. Ma, "Multiple positive solutions for nonlinear m-point boundary value problems," Applied Mathematics and Computation, vol. 148, no. 1, pp. 249-262, 2004.

[28] V. Lakshmikantham, S. Leela, and J. V. Devi, Theory of Fractional Dynamic Systems, Cambridge Academic, Cambridge, UK, 2009.

[29] D. R. Smart, Fixed Point Theorems, Cambridge University Press, Cambridge, UK, 1980. 


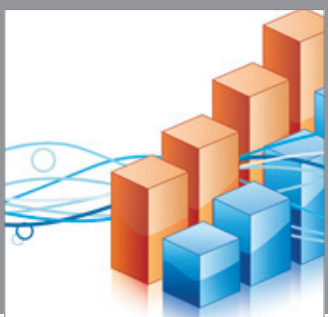

Advances in

Operations Research

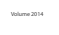

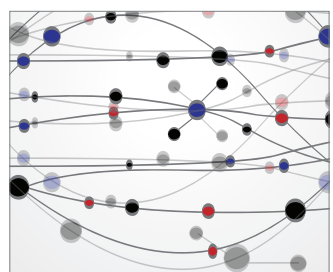

\section{The Scientific} World Journal
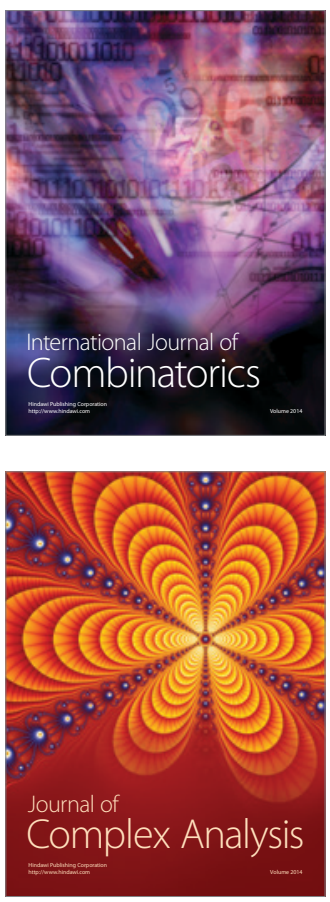

International Journal of

Mathematics and

Mathematical

Sciences
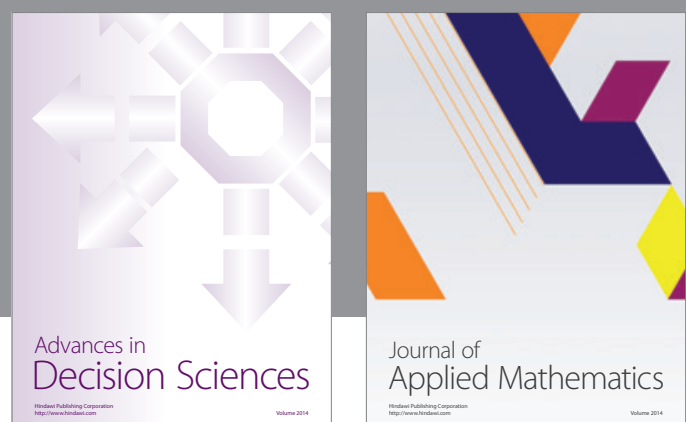

Journal of

Applied Mathematics
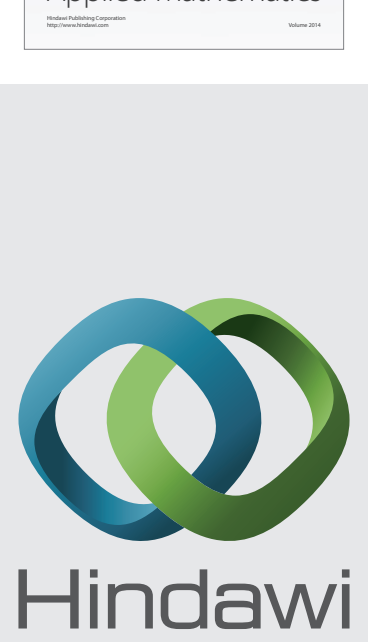

Submit your manuscripts at http://www.hindawi.com
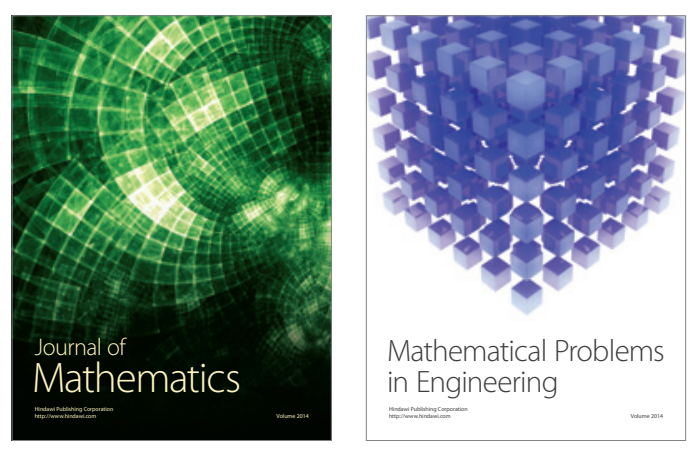

Mathematical Problems in Engineering
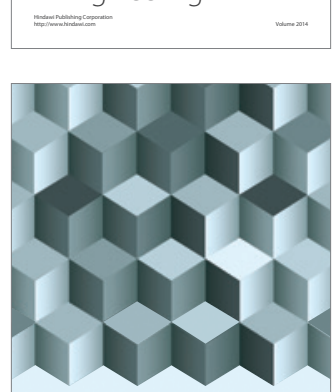

Journal of

Function Spaces
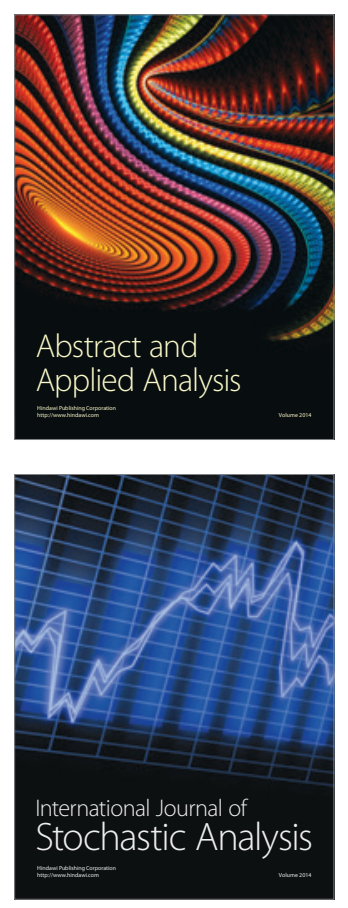

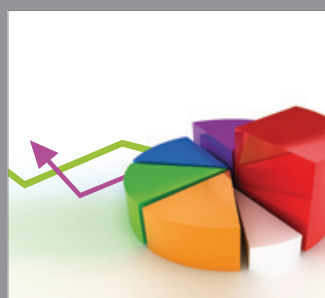

ournal of

Probability and Statistics

Promensencen
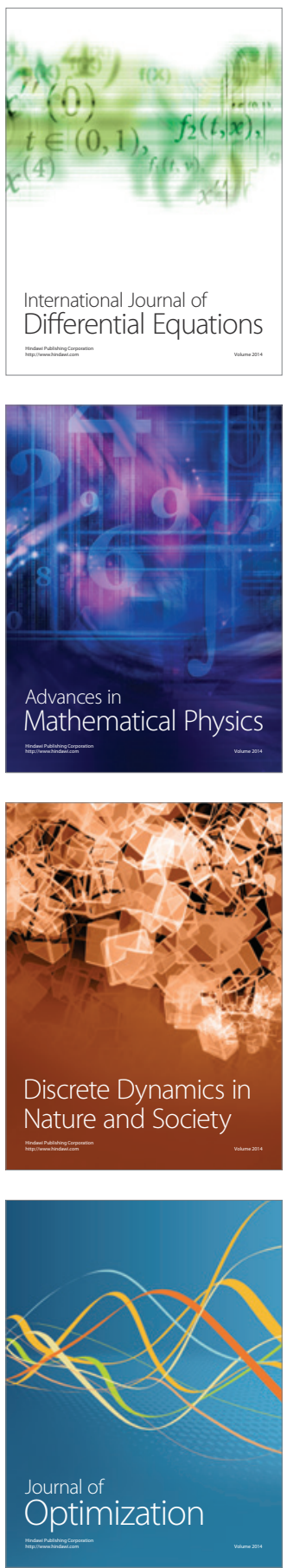